\title{
Unusual forms of venous thrombosis and thrombophilia
}

\author{
Ida Martinelli, MD, PhD \\ Angelo Bianchi Bonomi Hemophilia and Thrombosis Center, IRCCS Maggiore Hospital, University of Milan, Italy
}

\section{Key Words}

Venous thrombosis, thrombophilia, risk factors.

\begin{abstract}
Venous thromboembolism (VTE) results from multiple interactions between inherited and environmental risk factors. The lower limbs are the most common site of VTE, but more rarely other venous sites can be involved. The role of risk factors for VTE can be different in the various thrombotic manifestations, and there are specific risk factors for specific sites. Coagulation abnormalities causing inherited thrombophilia are frequently found in patients with cerebral vein thrombosis, but are more rare in those with "isolated" pulmonary embolism, upper limb or retinal vein thrombosis. Transient situations, such as surgery, trauma, prolonged immobilization, the use of oral contraceptives or hormone replacement therapy, and pregnancy or puerperium, are often recognized in patients with lower limb deep vein thrombosis, "isolated" pulmonary embolism, abdominal and cerebral vein thrombosis, but not in patients with upper limb deep vein thrombosis. Major risk factors for deep vein thrombosis of the upper limbs are strong efforts with the arms, whereas for abdominal vein thrombosis are myeloproliferative disorders and
\end{abstract}

liver cirrhosis In conclusion, there is increasing evidence that inherited and environmental risk factors may interact differently in determining VTE in different sites.

Copyright @ 2002 S. Karger AG. Basel

Venous thromboembolism (VTE) is a complex disease which results from multiple interactions between inherited and acquired risk factors (1). The most common manifestations of VTE are deep vein thrombosis of the lower limbs and its complication pulmonary embolism. Inherited determinants of VTE are only in part known, and are the deficiencies of the naturally occurring anticoagulant proteins antithrombin, protein $\mathrm{C}$ and protein $\mathrm{S}$, known since many decades, and the most recently discovered point mutations in genes coding for factor V (factor V Leiden or factor V G1691A) and factor II (prothrombin G20210A). Factor V Leiden and the prothrombin mutation are the most common inherited determinants of VTE, being present in approximately $20 \%$ and $10 \%$ of patients with VTE, respectively, and in $3 \%$ of the general population [1]. Inherited thrombophilia plays an important role in the etiology of lower limb deep vein thrombosis, being found in about onethird of patients with the disease. Other than inherited coagulation abnormalities, there are transient situations that are often described in association with VTE, such as cancer, recent surgery, trauma, prolonged immobilization, the use of oral contraceptives or hormone replacement therapy, and pregnancy or puerperium [1].

\section{KARGER \\ Fax +416130612 34

Dr. Ida Martinelli

Hemophilia and Thrombosis Center

IRCCS Maggiore Hospital

University of Milano - Via Pace, 9 - 20122 Milano - Italy

phone.:+39-02-5503.5468/5422 - fax: +39-02-50320723

e-mail: martin@policlinico.mi.it 
Other than the lower limbs, venous thrombosis can more rarely involve vascular districts such as the deep veins of the upper limbs, cerebral sinuses, retinal veins, and abdominal veins. Another rare manifestation of VTE is "isolated" pulmonary embolism, in which the source of emboli cannot be identified with objective techniques. The role of each risk factor may be different in the various thrombotic manifestation of VTE, and it appears that there are specific risk factors for specific sites.

\section{Upper limb deep vein thrombosis}

Studies on upper limb deep vein thrombosis (DVT) are less solid than those on lower limb DVT because of the rarity of this thrombotic manifestation. The most common cause of upper limb DVT is the insertion of indwelling catheters, that per se justifies thrombus formation. Studies on upper limb DVT not related to indwelling catheters have included small series of patients. It appears that thrombophilia is less frequent in upper limb than in lower limb DVT, with a prevalence of factor V Leiden lower than $10 \%$, less than half the prevalence reported in lower limb DVT [2,3]. Regarding transient risk factors, surgery, trauma or prolonged immobilization are rarely associated with the disease, and this can be at least in part explained by the absence of valves and therefore stasis in deep veins of the arms. A consistent result is that the so called "effort syndrome" is a peculiar and particularly frequent risk factor for upper limb DVT, being recognized in 20 to $40 \%$ of patients. The type of efforts reported are various, from rowing to weight lifting. Anatomical abnormalities, such as thoracic outlet compression or anomalous musculofascial bands, may aggravate the intrinsic compression of the veins during muscular activity of the arms. Such aggravation could cause endothelial damage, fibrosis around the vein, and reduction of blood flow, any of which could contribute to thrombus formation. Such anatomical abnormalities are often difficult to diagnose. It is of major importance after a first episode of upper limb DVT to advise the patient not to make excessive muscular efforts with his/her arms in the future; since upper limb DVT can embolize, it is necessary to give anticoagulant treatment similarly to that given in lower limb DVT (on average for 3 months).

\section{"Isolated" pulmonary embolism}

Pulmonary embolism (PE) is usually the complication of lower limb DVT. However, in approximately $20 \%$ of cases a source of emboli (deep and superficial veins of the limbs, abdominal veins, right cardiac chambers) cannot be identified even if searched carefully and with appropriate techniques. Where do emboli originate from in these cases? It is possible that thrombi generate locally in the pulmonary circulation, or that they detached completely from a peripheral vein not leaving any sign of their original presence. Apart from these speculation of the etiology of "isolated" PE, it has been clearly demonstrated that inherited thrombophilia is less frequent in these cases than in cases with lower limb DVT with or without PE (10\% or less for factor V Leiden). This observation derives from case-control studies that have obvious limitations and suffer from several possible bias, such as diagnostic bias. However, it was confirmed by some Authors who investigated for the presence of factor $\mathrm{V}$ Leiden an autoptic series of patients who had died for fatal PE [4]. At the moment, any pathophysiological consideration is merely speculative, and we can simply say that more accurate studies are needed to confirm this intriguing finding. The type and the prevalence of transient risk factors for "isolated" PE are similar to those reported for lower limb DVT: approximately 10-20\% are associated with surgery, trauma or prolonged immobilization, 40$50 \%$ with oral contraceptive use, and $20 \%$ with pregnancy or puerperium. The duration of oral anticoagulant treatment for a first episode of "isolated" PE does not differ from that of PE following DVT, i.e., 3 to 6 months.

\section{Cerebral vein thrombosis}

Cerebral vein thrombosis (CVT) is a frightening thrombotic manifestation because of the severity of symptoms and the high mortality rate (up to $30 \%$ in some series). CVT is a good model to investigate the role of genetic risk factors, since cerebral sinuses lack valves and stasis. Several case-control studies are available in the literature, consistently showing a high prevalence of thrombophilia in CVT cases, similar or higher than that found in patients with lower limb DVT (15-20\% for factor V Leiden). Among transient risk factors, a crucial role has the use of oral contraceptives (present at the time of CVT in up to $85-95 \%$ of women in some series) and also pregnancy and puerperium (30-80\%), explaining at least in part why the majority of the studies included a higher proportion of women than men [5]. How long patients with a first CVT should be treated with anticoagulants has never been assessed by specifically tailored studies; in clinical practice, warfarin is suggested for one year period and of course transient risk factors such as oral contraceptive use should be definitely removed.

\section{Abdominal vein thrombosis}

Data on thrombosis of the abdominal veins (portal vein, hepatic veins, mesenteric veins) are scanty. Recently two case control studies have been published, both considering separately hepatic and portal vein thrombosis, and both confirming an association between these thrombotic manifestations and 
thrombophilia [6,7]. For example, factor V Leiden was present in $3-8 \%$ of patients with portal vein thrombosis and in up to $26 \%$ of those with hepatic vein thrombosis. Another study carried out in 60 patients with portal vein thrombosis without preexisting liver cirrhosis found an overall $25 \%$ prevalence of thrombophilia [8]. These data need to be confirmed by further studies of larger sample size. The aforementioned studies included patients with strong risk factors for abdominal VT, such as myeloproliferative disorders (30-50\% of cases) and liver cirrhosis (15\% of cases). These diseases represent the strongest risk factors for abdominal VT. Others are surgery (20-30\% of cases) and oral contraceptive use (13-50\% of cases). Similarly to CVT, the duration of oral anticoagulant therapy in patients with abdominal VT is approximately one year, since this is considered a life-threatening event, with exceptions that are established on individual basis.

\section{Retinal vein occlusion}

Retinal vein occlusion (RVO) should not be included among unusual sites of VTE, since it is a relatively frequent disease, although its true prevalence remains unknown. There are various types of RVO depending on the site of occlusion (branch RVO, central RVO, hemicentral RVO), the most frequent of which is central RVO. RVO is a common complication of various systemic diseases including arterial hypertension, diabetes mellitus, dyslipidemia, and systemic vasculitis. Central RVO is also a complication of open-angle glaucoma or ocular hypertension; it is very rare in the young, among whom the precipitating factor is local inflammation. The role of thrombophilia in the etiology of RVO is marginal, whereas of primary importance are stasis and endothelial damage, the former due to the anatomical course of the vessels and the latter due to the compression changes induced by the lamina cribrosa. Hypercoagulability due to coagulation abnormalities has been investigated by a limited number of studies with small series of patients, that gave contrasting results [reviewed in 9]. Treatment for RVO consists mainly of managing any identified underlying systemic disease, since there is no specific therapy for RVO. If hypercoagulability due to thrombophilia is identified, there would be a rationale for the use of anticoagulant drugs, although such approach is not evidencebased and no demonstration of their utility exists.

\section{Conclusions}

The large number of studies carried out in the last decade after the discovery of the most common causes of thrombophilia, i.e., factor V Leiden and the prothrombin mutation, and the studies on the complex role of endothelium, greatly broadened our understanding on the etio-pathogenic mechanisms leading to thrombosis. The theory that thrombosis is an unique systemic disorder is no longer valid, and we have begun to appreciate the peculiar nature of the hemostatic balance of each vascular bed, through the knowledge of molecular mechanisms involved in blood coagulation and of the interaction between genetic and acquired risk factors (10). Future molecular and epidemiological research will consent to optimize patients' care.

\section{References}

1. Martinelli I: Risk factors for venous thromboembolism. Thromb Haemost 2001;86:395403

2. Martinelli I, Cattaneo M, Panzeri D, Taioli E, Mannucci PM: Risk factors for deep venous thrombosis of the upper extremities. Ann Intern Med 1997;126:707-11

3. Prandoni P, Polistena P, Bernardi E, Cogo A, Casara D, Verlato F, Angelini F, Simioni P, Signorini GP, Benedetti L, Girolami A: Upperextremity deep vein thrombosis. Risk factors, diagnosis, and complications. Arch Intern Med 1997; 157:57-62

4. Vandenbroucke JP, Bertina RM, Holmes ZR, Spaargaren C, van Krieken JHJM, Manten B, Reitsma PH: Factor V Leiden and fatal pulmonary embolism. Thromb Haemost 1998;79:511-6
5. Martinelli I, Sacchi E, Landi G, Taioli E, Duca F, Mannucci PM: High risk of cerebral-vein thrombosis in carriers of a prothrombin gene mutation and in users of oral contraceptives. $\mathrm{N}$ Engl J Med 1998;338:1793-7

6. Denninger $\mathrm{MH}$, Chait $\mathrm{Y}$, Casadevall N, Hillaire S, Guillin MC, Bezeaud A, Erlinger S, Briere J, Valla D: Cause of portal or hepatic venous thrombosis in adults: the role of multiple concurrent risk factors. Hepatology 2000;31:587-91

7. Janssen HL, Meinardi JR, Vleggar FP, van Uum SHM, Haagsma EB, van der Meer FJM, van Hattum J, Chamuleau RAFM, Adang RP, Vandenbroucke JP, van Hoek B, Rosendaal FR: Factor V Leiden mutation, prothrombin gene mutation, and deficiencies in coagulation inhibitors associated with Budd-Chiari syn- drome and portal vein thrombosis: results of a case-control study. Blood 2000;96:2364-8

8. Primignani $M$, De Bortoli N, Moia M, Bucciarelli P, Preatoni P, Carnevale P, de Franchis R: Extra-hepatic portal vein obstruction in adult patients: role of congenital or acquired thrombophilic disorders as etiologic factors. J Hepatol 2000; 32, Suppl 2, p 137 (abstract)

9. Prisco D, Marcucci R, Bertini L, Gori AM. Cardiovascular and thrombophilic risk factors for central retinal vein occlusion. Eur J Intern Med 2002;13:163-9

10. Rosenberg RD, Aird WC. Vascular-bed-specific hemostasis and hypercoagulable states. N Engl J Med 1999;340:1555-64 